\title{
Support Vector Regression Method for Regional Economic Mid- and Long-Term Predictions Based on Wireless Network Communication
}

\author{
Lingyu Dong $\mathbb{D}$ \\ School of Business Administration, Liaoning Technical University, Fuxin, 123000 Liaoning, China \\ Correspondence should be addressed to Lingyu Dong; 1600202108@sdtbu.edu.cn
}

Received 3 August 2021; Revised 1 September 2021; Accepted 6 September 2021; Published 22 September 2021

Academic Editor: Zhihan Lv

Copyright (c) 2021 Lingyu Dong. This is an open access article distributed under the Creative Commons Attribution License, which permits unrestricted use, distribution, and reproduction in any medium, provided the original work is properly cited.

\begin{abstract}
In recent years, wireless sensor network technology has continued to develop, and it has become one of the research hotspots in the information field. People have higher and higher requirements for the communication rate and network coverage of the communication network, which also makes the problems of limited wireless mobile communication network coverage and insufficient wireless resource utilization efficiency become increasingly prominent. This article is aimed at studying a support vector regression method for long-term prediction in the context of wireless network communication and applying the method to regional economy. This article uses the contrast experiment method and the space occupancy rate algorithm, combined with the vector regression algorithm of machine learning. Research on the laws of machine learning under the premise of less sample data solves the problem of the lack of a unified framework that can be referred to in machine learning with limited samples. The experimental results show that the distance between AP1 and AP2 is $0.4 \mathrm{~m}$, and the distance between AP2 and Client2 is $0.6 \mathrm{~m}$. When BPSK is used for OFDM modulation, $2500 \mathrm{MHz}$ is used as the USRP center frequency, and $0.5 \mathrm{MHz}$ is used as the USRP bandwidth; AP1 can send data packets. The length is 100 bytes, the number of sent data packets is 100 , the gain of Client2 is $0-38$, the receiving gain of AP2 is 0 , and the receiving gain of AP1 is 19 . The support vector regression method based on wireless network communication for regional economic mid- and long-term predictions was completed well.
\end{abstract}

\section{Introduction}

The wireless sensor network (WSN) is now a research field that has attracted widespread attention. With the rapid development of technologies such as microelectromechanical systems, chip systems, wireless communication systems, and integrated low-power systems, wireless sensor network technology has developed rapidly. Wireless sensor networks are widely used in daily life, military, industry, and many other fields to help people understand or master the real world more easily and quickly. Research on wireless sensor networks can be traced back to the 1970s. Initially, it was mainly used in the field of military research by the United States. In 1978, the Defense Advanced Research Projects Agency (DARPA) and Carnegie-Mellon University (Carnegie-Mellon University) jointly researched distributed sensor networks and held a seminar on distributed wireless sensor networks. The Sensor Webs project was researched by the National Space Administration (NASA) laboratory in 2001 to provide synchronized, all-weather, and interconnected global images through satellite sensors orbiting the earth to achieve rapid response to abnormal events. The technology is ready to be used in the field of Mars exploration. In recent years, my country has also strongly supported the research of wireless sensor networks. The "National Science and Technology Medium and Long-term Development Plan" includes "intelligent technology" and "network self-organizing technology" related to wireless sensor network research.

Once the wireless sensor network is developed, various detailed environmental data or target information can be obtained in the area of the detected object, so as to realize people's unpleasant or inaccessible perception of the physical world, so as to achieve communication between people. Wireless sensor networks have very wide application 
potential in many fields such as daily life, military, and industry. In daily life, it is used for medical treatment and health monitoring; for example, doctors can remotely collect various vital signs of patients, for environmental science, such as migratory bird migration, ocean, and earthquake monitoring. Due to its complexity, it is used in the military. In a war environment, collecting information through sensor nodes has become an indispensable part of the military field, providing surveillance, reconnaissance, and deployment functions for military command. Used in industrial fields, such as dangerous working environment or complex mechanical equipment and feedback the occupational health status of the monitoring area at any time. In the future development, wireless sensor networks will be more widely and meticulously applied to various fields of production and life.

In recent years, many scholars have put forward constructive opinions on this issue. Raza et al. proposed a general learning algorithm SVM (support vector machine) based on statistical learning theory. The algorithm is based on the theory of structural risk minimization and generalized error bounds and strikes a balance between empirical risk and confidence risk. But their research did not map the data samples in the low-dimensional space to the highdimensional space nor could it provide a way to solve some linear inseparable problems [1]. Tang and Wang thoroughly studied the generalization performance of support vector machines and extended their application areas to multiclassification and regression problems. But their research did not propose a variant algorithm of SVM, which does not have much reference value [2]. Bertini et al. gave a similar error bound for soft-margin support vector machines in the case of classification and regression. A support vector machine is a binary classification model. Its basic model is a linear classifier with the largest interval defined in the feature space. The largest interval makes it different from the perceptron. However, the margin of error in his research is still too large to be practical for promotion [3]. Shrestha and other scholars obtained a simplified support vector machine model by reducing the size of the support vector set within a limited loss boundary. This method has some shortcomings: first of all, it has a large amount of calculation, and it still simplifies after finding all the support vectors, and while simplifying, because it is an indiscriminate reduction, the accuracy of recognition is sacrificed [4]. After in-depth research on the basis of RSVM (Reduced Support Vector Machine) proposed by the predecessors, Borisov and Pudalov artificially restricted the support vector to a subset containing training samples. Obviously, this method can reduce the support vector only when the number of training samples is large and the support vector occupies a very high proportion of it. In general, the number of support vector is will not only do not decrease but may increase [5]. In order to reduce the QSSVM algorithm's one-sided pursuit of iterative update times and the need to repeatedly solve the problem of quadratic programming, Liu et al. used the average Euclidean distance method to quickly optimize the QSSVM model, but his research did not use the support vector data description algorithm for outlier detection, which makes the algorithm process complicated, and there is no training sample reduction strategy [6]. Purnima et al. proposed an incremental hyperellipsoid model for the unsteady distribution of sensor network data streams. The detected outliers are used to trigger the incremental update of the decisionmaking model. The algorithm achieves nearly real-time tracking of the data stream. But their research did not reduce the time complexity of the kernel function calculation [7].

The innovations of this paper are the following: (1) The design of the system data source is proposed, the scale of the data source of the decision-making system and the parameter type of the vector machine are selected, and the CC2530 single-chip microcomputer is innovatively selected as the system carrier. (2) The boundary of generalization error is deduced, and the algorithm of inequality is given to describe the relationship between the empirical risk and the actual risk of function concentration. (3) In the discussion part, the introduction of the vector regression method is proposed, and the introduction is from the shallow to the deep from the perspective of mechanical learning, which strengthens the logic of the article.

\section{Support Vector Regression Method for Medium- and Long-Term Predictions of Regional Economy Based on Wireless Network Communication}

2.1. Definition and Classification of Wireless Network Sensors. In practical applications, the wireless sensor network system is a hierarchical application structure model. The following mainly introduces the overall architecture design and operation of each level of the wireless network sensor application system [8]. Aiming at the shortcomings of the single-gateway system structure of the wireless sensor network, a multigateway transmission system is proposed [9]. The gateway is essentially an IP address from a network to other networks. For example, there are network $\mathrm{A}$ and network B. The IP address range of network $\mathrm{A}$ is 192.168.1.1 to 192.68.1.254, and the subnet mask is 255.255.255.0; the IP address range of network $B$ is "192.168.2.1 to 192.168.2.254." The netmask is 255.255.255.0. In the absence of a router, TCP/IP communication cannot be carried out between the two networks, even if the two networks are connected to the same switch (or hub), the TCP/IP protocol is also unable to communicate. According to the subnet mask, it is determined that the hosts in the two networks are in different networks, as shown in Figure 1.

In modern wireless local area networks [10], the development of dense wireless access points makes adjacent cofrequency networks more and more common [11, 12]. The use of the same channel in adjacent networks makes the channel competition of each node in the network fierce with serious interference $[13,14]$. The transmission efficiency drops sharply, which seriously affects the transmission performance of the entire network [15]. Network congestion (congestion) refers to the situation wherein when too many packets are transmitted in the packet switching 


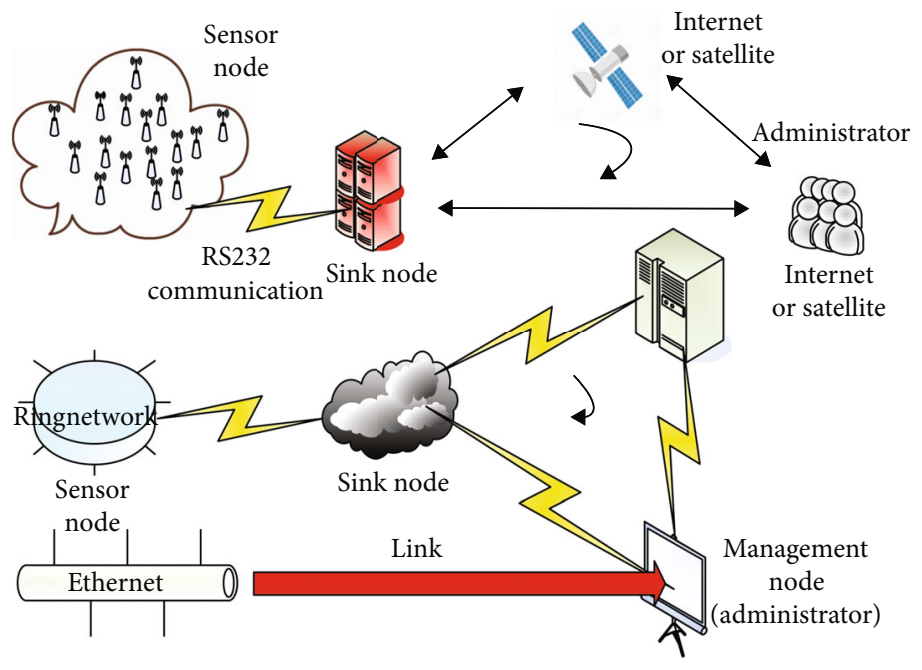

FIGURE 1: Wireless sensor network structure.

network, the network transmission performance is reduced due to the limited resources of the store and forward nodes. When the network is congested, data loss generally occurs, the delay increases, and the throughput decreases. In severe cases, it may even lead to "congestion collapse" (congestion collapse). Under normal circumstances, network congestion occurs when the network performance is degraded due to excessive increase in the load on the network. In order to improve the efficient performance of business wireless LAN transmission, simultaneous multinode communication technology on the network has become a current research hotspot $[16,17]$. The modern multinode communication technology studied today is mainly to combine and synchronize access points with wireless local area networks to form a huge distributed multiantenna system $[18,19]$. This requires high synchronization accuracy between different wireless access points, and simultaneous uplink communication or simultaneous downlink communication can be achieved at a specific time [20].

In real scenarios, it is not uncommon for a large amount of data to be transmitted up and down between adjacent subnets [21], but there is a lack of research on simultaneous uplink and downlink communication technologies [22]. Based on the characteristics of wireless local area networks and the coexistence of single-antenna nodes and multiantenna nodes on the client side in real life [23], this work establishes a system model and uses the advantages of the central controller to achieve synchronous communication [24]. Two interference cancellation techniques are used for the interference and interference problems on the uplink and downlink AP sides, namely, interference reset and coordinated interference cancellation, so that the receiving node can receive data packets correctly [25]. Cooperative interference cancellation is to estimate the interference introduced by different users and multipaths and then subtract the interference estimate from the received signal (the judge must first filter out the influence of various interferences when receiving a case). Serial Interference Cancellation (SIC) is to gradually subtract the largest user interference, which is equivalent to a case being judged by different judges successively; Parallel Interference Cancellation (PIC) is to simultaneously reduce and remove the interference of all other users outside of itself, which is equivalent to multiple judges trying the same case at the same time. It certainly saves time to make a judgment at the same time than to make multiple judgments one after the other. In the same way, the signal processing delay of PIC is more reduced than that of SIC. The functional structure of the sensor is shown in Figure 2.

\subsection{Focus of SVM Improvement and Perfection}

2.2.1. Increase Training Speed. In general, the larger the number, the better the learning effect, but more support vectors will increase the calculation response of the learner. Therefore, if you want to increase the computing speed of SVM and make it more calmly face large-scale data processing problems, it is very meaningful to reduce the number of support vectors. Another idea to speed up the classification of support vector machines is to replace all support vectors with a small amount of support vectors and obtain a sparse representation of the existing support vectors. The disadvantage of this method is that solving the self-built transformation matrix of reduced support vectors is a more complex optimization problem. Using some numerical calculation software, the value that satisfies the optimal result can be calculated on the training data set and then solved. Obviously, the points on the support vector satisfy the Slater condition, so the optimization problem satisfies the Slater condition, so the solution of the dual problem is equivalent to the solution of the original problem, so that we can directly solve the optimal solution of the dual problem. There are also some algorithms that can quickly calculate the results. We will introduce these later. The construction of the support vector machine in the case of completely linear separability is completed here. In fact, not all values are nonzero; only the point on the support vector machine has a nonzero value, which is consistent with the first case in the inequality constraint. 


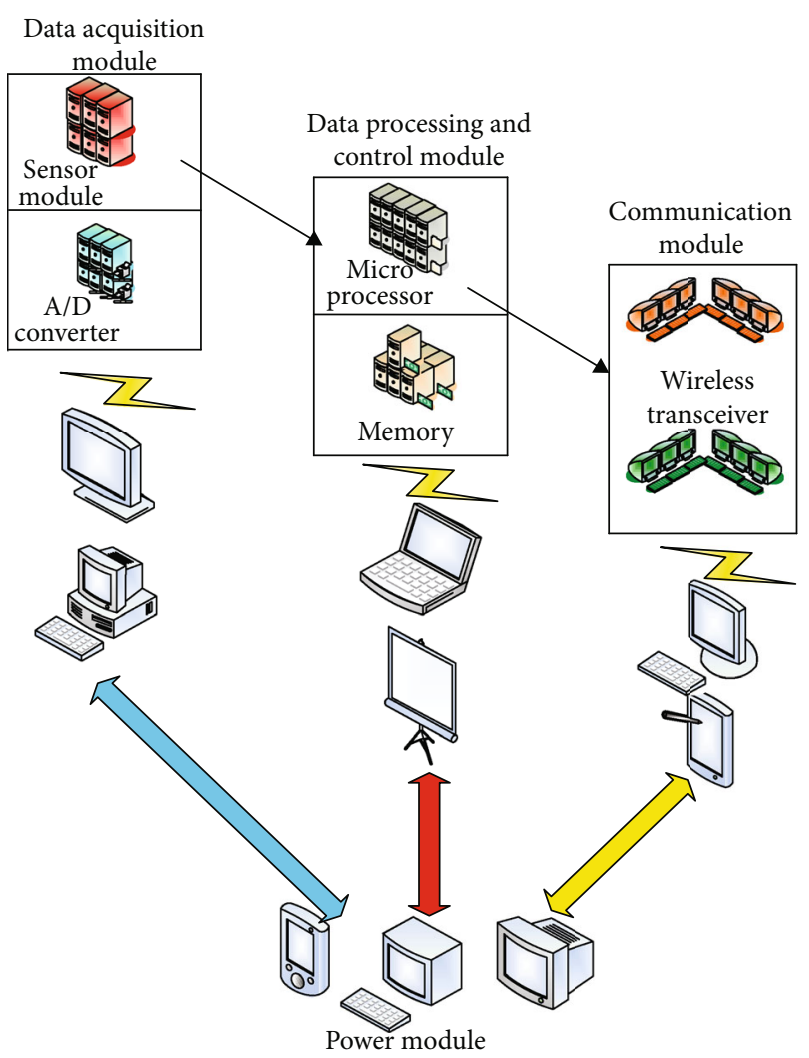

FIgURE 2: Functional structure of the sensor.

2.2.2. Research on Support Vector Machine Multiclassification Algorithm. Based on the problem prototypes in real life, the modeling of many problems needs to be explained from the perspective of multicategory. The classic SVM algorithm has achieved good results in the study of two types of classification problems and has been widely used. How to effectively transfer the more mature two classification capabilities of SVM to multiclass classification problems is a practical requirement for expanding the application field of support vector machines' high research value. SVM is developed from the optimal classification surface in the case of linear separability. The optimal classification surface requires that the classification line not only correctly separates the two classes (training error rate is 0 ) but also maximizes the classification interval. SVM considers looking for a hyperplane that meets the classification requirements and makes the points in the training set as far as possible from the classification surface, that is, looking for a classification surface to maximize the margin on both sides of it. The training samples of $\mathrm{H} 1$ and $\mathrm{H} 2$ on the hyperplane that is closest to the classification surface and parallel to the optimal classification surface in these two types of samples are called support vectors.

2.2.3. Optimization of Learning Problems. The optimization of the algorithm is mainly reflected in the improvement of the generalization ability of the algorithm and the solution of the problems encountered in the actual application of the algorithm. When training the SVM learner with samples, if the positive and negative samples are highly mixed, the phenomenon of over-learning may occur, which makes the classification surface too complex and over-loyal to the sample set, which reduces the generalization ability. In addition, the processing of approximate outliers and noise existing in the sample, as far as possible, to eliminate the impact of these two types of data on the classification results is also the focus of research. Based on this problem, fuzzy concepts with strong robustness are introduced into SVM research, such as the F-SVM algorithm. The comparison table of the difference and connection between the two algorithms of SVM and F-SVM is shown in Table 1.

2.2.4. Improvement of SVR (Support Vector Regression) Algorithm. Classification and regression problems are the main problems to be solved in machine learning methods. With the continuous emergence of classification algorithms, the use of support vector machines for regression learning on nonlinear problems has entered people's field of vision, and its structural risk based on statistical theory is minimized. The criterion makes it have certain advantages in the process of function optimization.

2.3. Space Occupancy Rate. The space full rate refers to the ratio of the memory occupied by all bytes to the total capacity of the path to be tested in a given period of time. The data is analyzed through the dimensions of traffic density, reflecting the occupancy of the line to be detected at a given time. However, because the data cannot be obtained directly, it is not applicable in most cases.

$$
R_{s}=\frac{1}{2} \sum_{i=1}^{n} l_{i} .
$$

The formula for time occupancy is

$$
R_{t}=\frac{1}{T} \sum_{i=1}^{n} t_{i} .
$$

Historical average method: the average value of the data at the same time on different dates is used as the estimated value of the missing value, as shown in

$$
y(t)=\frac{1}{n} \sum_{i=1}^{n} y_{i}(t) .
$$

Adjacent data averaging method: the average value of adjacent moments is used as the estimated value, as shown in

$$
y(t)=\frac{y(t-1)+y(t+1)}{2} .
$$

Weighted average method: use the weighted average of adjacent historical data and adjacent actual measured values as the estimated value. The weighted average method uses several past observations of the same variable arranged in chronological order and uses the number of times the 
TABLE 1: Comparison of the two algorithms of SVM and F-SVM.

\begin{tabular}{lc}
\hline SVM & F-SVM \\
\hline Classification algorithm & Classification algorithm \\
Line type classification & Kernel function \\
Nonscaling invariance & Scalability invariance \\
\hline
\end{tabular}

time-sequential variable appears as the weight to calculate the weighted arithmetic average of the observations, and this number is used as the forecast for the future period. A trend forecasting method for the predicted value of a variable, as shown in

$$
y(t)=y \gamma(t-1)+(1-\gamma) y_{i}(t)
$$

When using this function as a filter, the frequency band correlation is relatively large, which is convenient for filtering processing. The wavelet function is

$$
\psi(t)=\sqrt{2} \sum_{k \in z}(-1)^{1-k} \bar{h}_{k} \varphi(2 t-k) k \in z
$$

Using linear function conversion, the data is normalized between 0 and 1 , as the statistical probability distribution. The specific formula is as follows:

$$
y=\frac{x-\text { MinValue }}{\text { MaxValue }- \text { MinValue }} .
$$

Among them, MaxValue is the maximum value of the training sample set and MinValue is the minimum value. In the field of machine learning, different evaluation indicators (that is, the different features in the feature vector are the different evaluation indicators) often have different dimensions and dimensional units. This situation will affect the results of data analysis. In order to eliminate the indicators, the dimensional influence between the data needs to be standardized to solve the comparability between data indicators. After the raw data is processed by data standardization, the indicators are in the same order of magnitude, which is suitable for comprehensive comparative evaluation. Among them, the most typical is the normalization of data. The existence of a singular sample data will increase the training time and may also lead to failure to converge. Therefore, when there is singular sample data, it is necessary to normalize the preprocessed data before training; conversely, when there is no singular sample data, then normalization is not required. If the normalization is not performed, the value of different features in the feature vector is quite different, which will cause the objective function to become "flat." In this way, when performing gradient descent, the direction of the gradient will deviate from the direction of the minimum value and take many detours, that is, the training time is too long.

Next, we will introduce the algorithm of vector machine mechanical learning.

Statistical learning theory (SLT) has a solid theoretical foundation. The difference from traditional statistics is that the study of the laws of machine learning under the premise of less sample data solves the problem of the lack of a unified framework for machine learning with limited samples.

The problems to be studied in machine learning are as follows: $n$ independent samples are known:

$$
\left(x_{1}, y_{1}\right),\left(x_{2}, y_{2}\right), \cdots,\left(x_{n}, y_{n}\right)
$$

Solve for an $f$ that minimizes the expected risk:

$$
R(w)=\int L(y, f(x, w)) d F(x, y)
$$

When predicting $y$, it will show different forms depending on the type of learning problem. The loss function can be defined as

$$
L(t, f(x w))=\left\{\begin{array}{ll}
0, & \text { if } y=f(x, w) \\
1, & \text { if } y \neq f(x, w)
\end{array}\right\}
$$

The learning problem is that when the sample is known but the probability density $F(x, y)$ is unknown, finding a $f($ $x, w 0)$ can minimize the error probability of classification, that is, minimize the risk functional.

In the function regression estimation problem, the loss function is

$$
L(y, f(x, w))=(y-f(x, w))^{2}
$$

In order to pursue the machine learning to minimize the experience risk, since the probability density $F(x, y)$ is unknown, the sample error is usually used as an indicator to measure the experience risk, that is, the experience risk minimization criterion is adopted:

$$
R_{\mathrm{emp}}(w)=\frac{1}{l} \sum_{i=1}^{1} L\left(y_{i}, f\left(x_{i}, w\right)\right) .
$$

An important concept in SLT is the VC dimension, which derives the boundary of generalization error and gives the following inequality to describe the relationship between the empirical risk and actual risk of function concentration, that is, the generalized boundary:

$$
\begin{gathered}
R(a)<R_{\mathrm{emp}}(a)+\psi\left(\frac{h}{l}\right), \\
\psi\left(\frac{h}{l}\right)=\sqrt{\frac{h((\ln (2 l / h))+1)-\ln (n / 4)}{1} .}
\end{gathered}
$$

Therefore, the optimization problem is solved as follows:

$$
\left\{\begin{array}{l}
\min \frac{1}{2}\|w\|^{2}, \\
\text { s.t. } y_{i}\left(<w, x_{i}>+b\right)-1 \geq 0, \quad i=(1,2, \cdots, N) .
\end{array}\right.
$$


(a) Star network $\mathrm{O}$
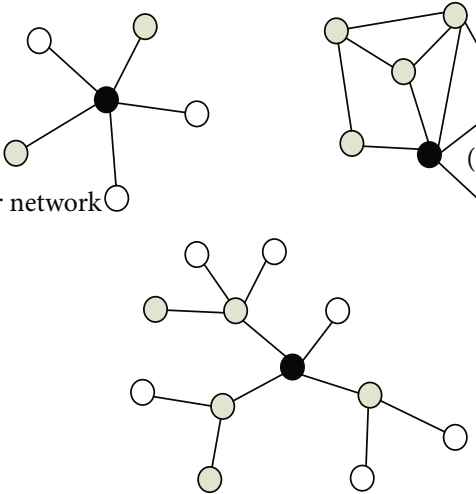

(b) Tree network

Coordinator node

Coordinator node

Terminal node

FIGURE 3: Zigbee protocol topology.

\section{Experiment and Analysis of the Support Vector Regression Method Based on Wireless Network Communication for Regional Economic Mid- and Long-Term Predictions}

3.1. Zigbee Wireless Sensor Network Design. In the Ethernet, when a host communicates directly with another host, it is necessary to know the MAC address of the target host (because MAC is the only identifier of the real host). For example, "frames" are actually transmitted in the local area network, and the frame contains the MAC address of the target host. Since data transmission relies on the MAC address instead of the IP address, the ARP protocol must be used to convert a known IP address to a MAC address. "Address resolution" is the process by which the host converts the target IP address into the target MAC address before sending the frame. The basic function of the ARP protocol is to query the MAC address of the target device through the IP address of the target device to ensure smooth communication. There are many transmission protocols for implementing wireless sensor networks, and the underlying network protocol used in the wireless sensor network designed in this article is the Zigbee protocol. It is a series of communication protocols developed by the Zigbee Alliance to achieve low data rate and short-distance wireless transmission requirements [26-29]. The Zigbee protocol also uses a multilevel network structure model, and data communication is carried out between the protocol stack levels through the service access point (SAP). There are two interfaces between most levels: data service interface and management service interface [30]. The topological structure of Zigbee agreement is shown as in Figure 3.

It can be seen from the above analysis that the star network has a simple structure and is suitable for occasions with small network nodes. The tree network is an extension of the star network. Its network scale is larger than that of the star network. Nodes can also communicate with each other, but they must be routed through the tree. If the rout-
TABLE 2: CC2530 main performance parameters.

\begin{tabular}{lc}
\hline Performance parameter & Index \\
\hline Frequency band & $2405 \sim 2480 \mathrm{MHz}$ \\
Main chip & CC2530F256 \\
Communication protocol standard & IEEE 802.15 .4 \\
Modulation & DSSS (O-QPSK) \\
Data transfer rate & $250 \mathrm{~kb} / \mathrm{s}$ \\
Communication range & Open occasion $400 \mathrm{~m}$ (max) \\
Acceptance sensitivity & $-97 \mathrm{dBm}$ \\
Addressing mode & 64 -bit IEEE address \\
Data encryption & $128 \mathrm{~b}$ AES \\
\hline
\end{tabular}

ing nodes in these two networks fail, it will likely lead to the paralysis of the entire network. In comparison, mesh network nodes are large in scale [31]. Adjacent routing nodes can directly communicate with each other without forwarding through the parent node, and remote nodes can also communicate with each other through relay nodes. It can be seen that the mesh network is more flexible and suitable for occasions where the network structure may change over time. However, the mesh network layout is relatively complicated, and because the routing nodes in the network need to maintain neighbor routing tables, routing forwarding tables, and routing discovery tables, routing nodes need a certain amount of memory overhead.

The Zigbee network node is realized by the CC2530 single-chip microcomputer. The main performance of the CC2530 RF module is shown in Table 2.

IEEE 802.15.4 is a technical standard that defines a lowrate wireless personal area network (LR-WPAN) protocol. It specifies the physical layer and media access control of LRWPAN and is maintained by the IEEE 802.15 working group, which defined the standard in 2003. It is the basis of Zigbee, such as ISA100.11a, WirelessHART, MiWi, 6LoWPAN, thread, and SNAP specifications, each of which extends the standard by developing upper layers that are not defined in IEEE 802.15.4. CC2530 includes a power management function in the design, which can realize a low-power operation mode that does not use the power supply mode to extend the service life of the battery. CC2530 has 5 different operating modes (power supply mode), namely, active mode, idle mode, PM1, PM2, and PM3. Active mode is a general mode, and PM3 has the lowest power consumption. The influence of different power supply modes on system operation is shown in Table 3 , and the choice of voltage regulator and oscillator is also given.

$\mathrm{KMO}$ value and Bartlett sphericity tests are performed. Before performing factor analysis on variables, $\mathrm{KMO}$ values need to be used to test whether the data is suitable for factor analysis. The value range is from 0 to 1 . The closer the value is to 1 , the more common factors are between the variables and the more suitable for factor analysis. The specific content is shown in Table 4.

The sig value refers to the significance (significance), which is also the $P$ value, and the statistical significance (sig) refers to the probability of the result in the current 
TABLE 3: CC2530 power supply mode.

\begin{tabular}{lccc}
\hline Power supply mode & High frequency oscillator & Low frequency oscillator & Voltage regulator (digital) \\
\hline Configuration & A: $32 \mathrm{MHz}$ crystal oscillator & C: $32 \mathrm{kHz}$ crystal oscillator & \\
Active/idle mode & B: $16 \mathrm{MHz}$ RC oscillator & D: $32 \mathrm{kHz}$ RC oscillator & On \\
PM1 & A or B & C or D & On \\
PM2 & No & C or D & Off \\
PM3 & No & C or D & Off \\
\hline
\end{tabular}

TABLE 4: KMO and Bartlett test results.

\begin{tabular}{|c|c|c|c|c|}
\hline Scale & $\mathrm{KMO}$ & Approximate chi-square & $\mathrm{df}$ & Sig \\
\hline Supply chain trust & 0.828 & 329.404 & 45 & $\leq 0.001$ \\
\hline Supply chain information sharing & 0.847 & 663.350 & 91 & $\leq 0.001$ \\
\hline Supply chain agility & 0.870 & 329.286 & 28 & $\leq 0.001$ \\
\hline
\end{tabular}

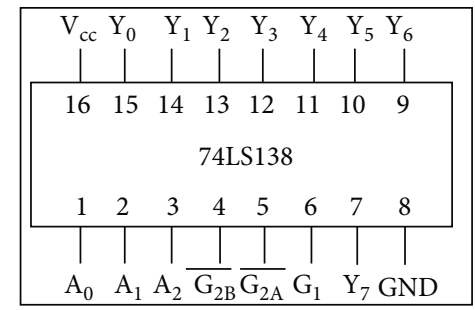

FIGURE 4: Single-chip pin drawing and function display.

TABle 5: Rotating component matrix of supply chain trust scale.

\begin{tabular}{lcc}
\hline Supply chain trust & \multicolumn{3}{c}{ Ingredient } \\
\hline CT1 & 1 & 2 \\
CT2 & 0.781 & 0.093 \\
CT3 & 0.732 & 0.207 \\
CT4 & 0.714 & 0.290 \\
CT5 & 0.686 & 0.269 \\
GT1 & 0.787 & 0.180 \\
GT2 & 0.238 & 0.600 \\
GT3 & 0.326 & 0.621 \\
GT4 & 0.048 & 0.682 \\
GT5 & 0.189 & 0.658 \\
\hline
\end{tabular}

sample. If the significance sig value is very small, such as < 0.05 (less than $5 \%$ probability), that is to say, if the overall "really" does not make a difference, then only when the chance is very small (5\%), which is very rare, will the current situation of this book appear. Although there is still a $5 \%$ chance of error, the following can be said with "relative confidence": this situation in the current sample is not a coincidence but is statistically significant. $\mathrm{df}$ is the degree of freedom, which refers to the number of independent or freely variable independent variables in the sample when the overall parameters are estimated by the sample statistics, which is called the degree of freedom of the statistics. The results of the analysis of variance in SPSS are shown in the
TABLE 6: W5500 and main controller pin connection table.

\begin{tabular}{lccc}
\hline $\begin{array}{l}\text { STM32F4 pin } \\
\text { number }\end{array}$ & $\begin{array}{c}\text { W5500 pin } \\
\text { number }\end{array}$ & $\begin{array}{c}\text { STM32F4 pin } \\
\text { number }\end{array}$ & $\begin{array}{c}\text { W5500 pin } \\
\text { number }\end{array}$ \\
\hline 40/PA4 & $32 / \mathrm{SCSn}$ & $43 / \mathrm{PA} 7$ & $35 / \mathrm{MOSI}$ \\
41/PA5 & $33 / \mathrm{SCLK}$ & $44 / \mathrm{PC} 4$ & $36 / \mathrm{INTn}$ \\
42/PA6 & $34 / \mathrm{MISO}$ & $45 / \mathrm{PC} 5$ & $37 / \mathrm{RSTn}$ \\
\hline
\end{tabular}

Figure 4, where df is the degree of freedom, $F$ is the $F$ value, and sig is the $P$ value. Exploratory factor analysis of the supply chain trust scale is as follows: after the software extracts two common factors, the total explained variance is 54.343 $\%>50 \%$. The rotation component matrix of the trust degree of the supply chain is shown in Table 5.

The pin connection table of W5500 and the main controller is shown in Table 6.

Protel99SE performs single-chip pin drawing and function display as shown in Figure 4.

3.2. Experimental Results. The experiment uses LS-SVMlab, which is a software package suitable for the MATLAB experiment toolbox, which can be applied to different computer operating systems. In the software package, the parameters of some functions are the experience parameters of the editors and researchers after experiments. It is more general, so some test parameters in the article are set based on experience on the basis of reference to the existing parameters of the program [32].

According to the learning method steps in the article, after normalizing the data, first use the LS-SVR learner to conduct preliminary training on the sample set, and the obtained prediction curve is shown in Figure 5.

Next, draw a graph of the results of the simulation experiment performed by MATLAB. The MIMO system (Multiple-Input Multiple-Output) refers to the use of multiple transmitting antennas and receiving antennas at the transmitting end and the receiving end, respectively, so that signals are transmitted and received through multiple antennas at the transmitting end and the receiving end, 


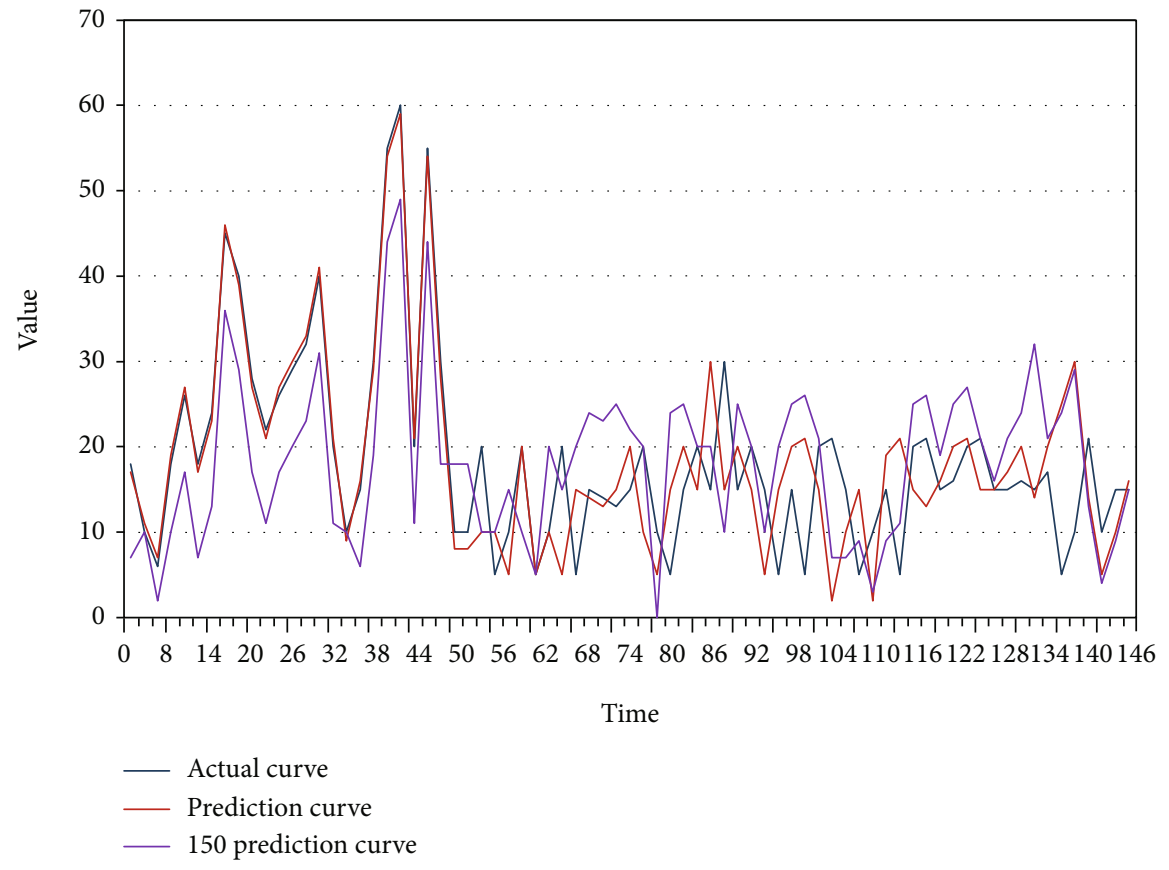

FIgURE 5: Prediction curve.

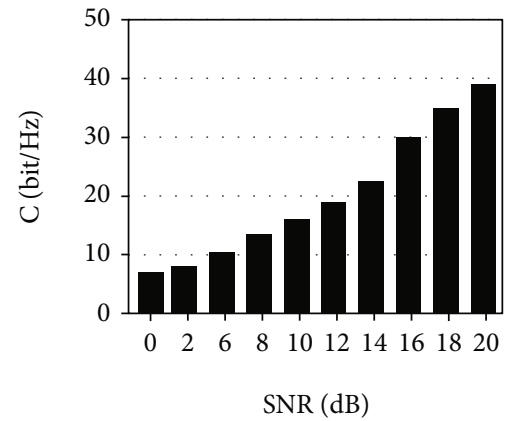

- $\mathrm{N}=\mathrm{M}=1$

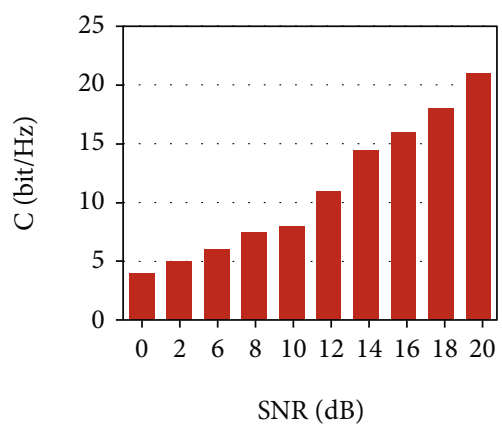

- $\mathrm{N}=\mathrm{M}=2$

Figure 6: Channel capacity of MIMO system with different antenna configurations (a).

thereby improving communication quality. It can make full use of space resources and achieve multiple transmissions and multiple receptions through multiple antennas. Without increasing spectrum resources and antenna transmission power, it can double the system channel capacity, showing obvious advantages and being regarded as the next generation of mobile core technology of communication. In the experiment, the channel capacity test of the MIMO system with the number of antennas $[1,2,4,8]$ was carried out. The SISO system with the number of antennas [1] can be regarded as a special case of the MIMO system. The situations of $N=M=1$ and $N=M=2$ are shown in Figure 6.

The situation of $N=M=4$ and $N=M 8$ is shown in Figure 7.

3.3. Performance Comparison of Algorithms. In order to evaluate the performance of the proposed hierarchical coverage area optimization algorithm based on the genetic algorithm (GACOA), we compare the performance of coverage area algorithms based on the virtual potential field (PFCEA) and the coverage area algorithm (CS). With regard to the practical application algorithm (PFCEA) and the CS algorithm, especially in the field of industrial control switching power supply, PID control technology has always been dominant. In recent years, the research and application of fuzzy control have been developed rapidly. When the uninterruptible power supply is working, there is instantaneous loading. When there are various types of loads, the PFC module uses PID algorithm compensation to often produce a certain degree of output oscillation. When the oscillation is frequent, it will seriously affect the service life of the PFC module. In this regard, adding the PFCEA and CS algorithms reduces the oscillation by adjusting the response ratio of the PID algorithm and improves the stability of the PFC module output and the adaptability of the load capacity. The number of nodes in the network is increased from 20 to 160 . In order to obtain accurate evaluation results, each simulation is performed 100 times, and the average value 

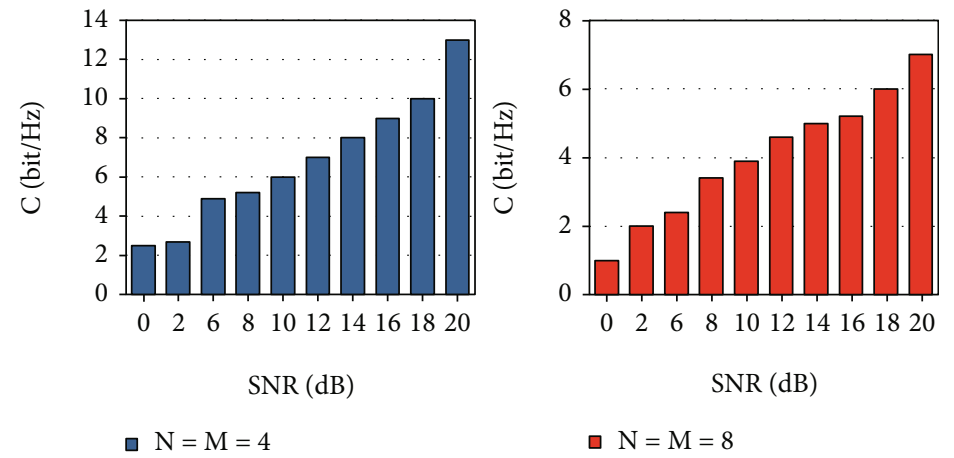

FIgURE 7: Channel capacity of MIMO system with different antenna configurations (b).
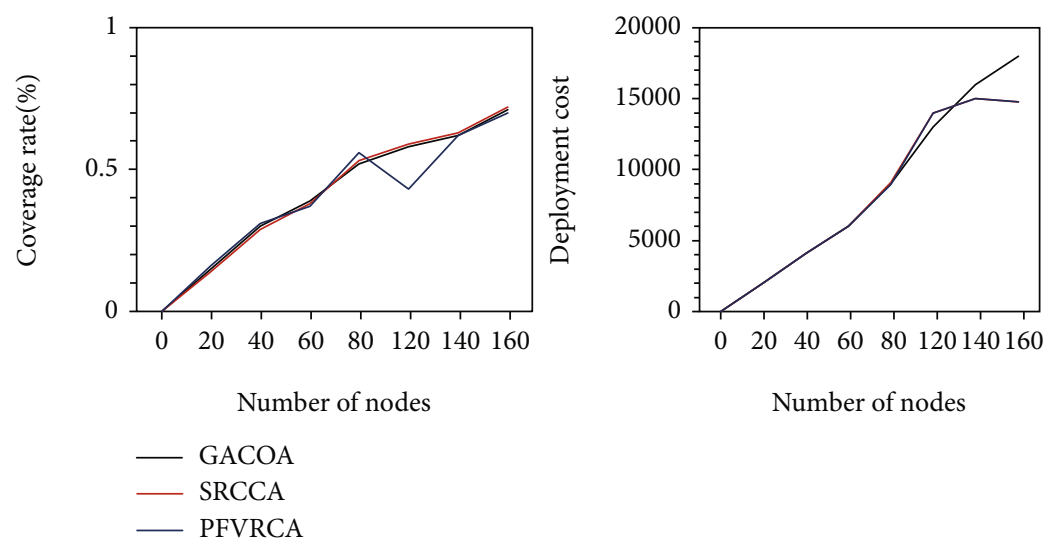

FIGURE 8: GACOA, PFCEA, and CSRCA performance comparison.

of each data is taken. The performance comparison of GACOA, PFCEA, and CSRCA is shown in Figure 8.

In order to evaluate the pros and cons of the threedimensional wireless ultraviolet ad hoc network networking strategy (UVNNS), the performance of UVNNS and the random deployment method (RDA) in the threedimensional wireless ultraviolet ad hoc network was compared. In the simulation experiment, each round of experiment is carried out 50 times, and the average value of each data is obtained, so as to obtain a more accurate conclusion. The algorithm performance comparison is shown in Figure 9.

When the transmitter transmits data under the condition of different gains, the correct rate of Client1 successfully receiving data packets from AP1 is shown in Figure 10 when Client2 does not interfere with Client 1 and interferes with Client1 and when interference is zeroed.

The success rate of AP1 receiving data packets from Client2 in different states is shown in Figure 11.

As shown in the figure, when AP2 adopts the cooperative interference cancellation technology, as the transmission gain of Client2 increases, the correct AP2 message download rate continues to increase, the gain increases to a certain extent, and the interference effect is not achieved. In the interrupt mode, even if the transmission gain of Client2 is set to the gain rate supported by USRP, the correct AP2 data packet download rate from Client2 is still 0 . The test shows that the interoperability interrupt cancellation technology between APs combined with the increase of client power can achieve correct reception of data packets in APs under simultaneous transmission. The distance between AP1 and $\mathrm{AP} 2$ is $0.4 \mathrm{~m}$, and the distance between AP2 and Client2 is $0.6 \mathrm{~m}$. When BPSK is used for OFDM modulation, $2500 \mathrm{MHz}$ is used as the USRP center frequency, $0.5 \mathrm{MHz}$ is used as the USRP bandwidth, and the length of the data packet that can be sent by AP1 is 100 bytes. The number of data packets sent is 100 , the gain of Client 2 is $0-38$, the receiving gain of AP2 is 0 , and the receiving gain of AP1 is 19.

The gain comparison of the two algorithms is shown in Figure 12.

\section{Discussion on the Support Vector Regression Method for Medium- and Long-Term Predictions of Regional Economy Based on Wireless Network Communication}

4.1. Matching Theory. The usual financial resource allocation mechanism is to use the market price mechanism to optimize resource allocation. Although some distribution problems in the real society can be solved through the price system, some problems in the price system will lead to social equity, such as the distribution of primary and secondary school students. Some distribution problems have not yet reached a fully competitive market process. In many cases, 


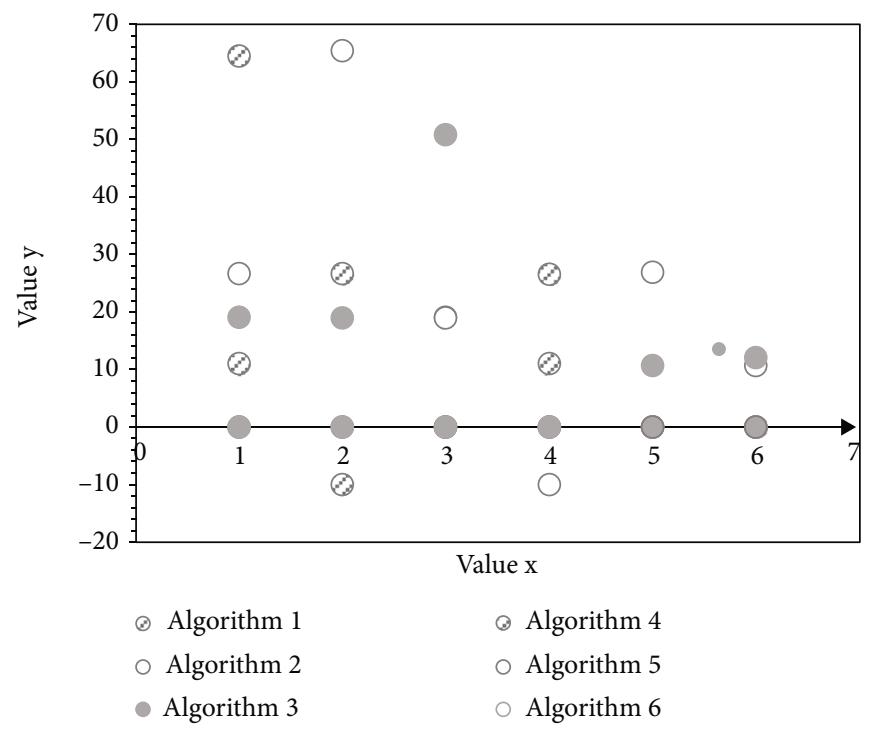

FIGURE 9: Algorithm performance comparison.

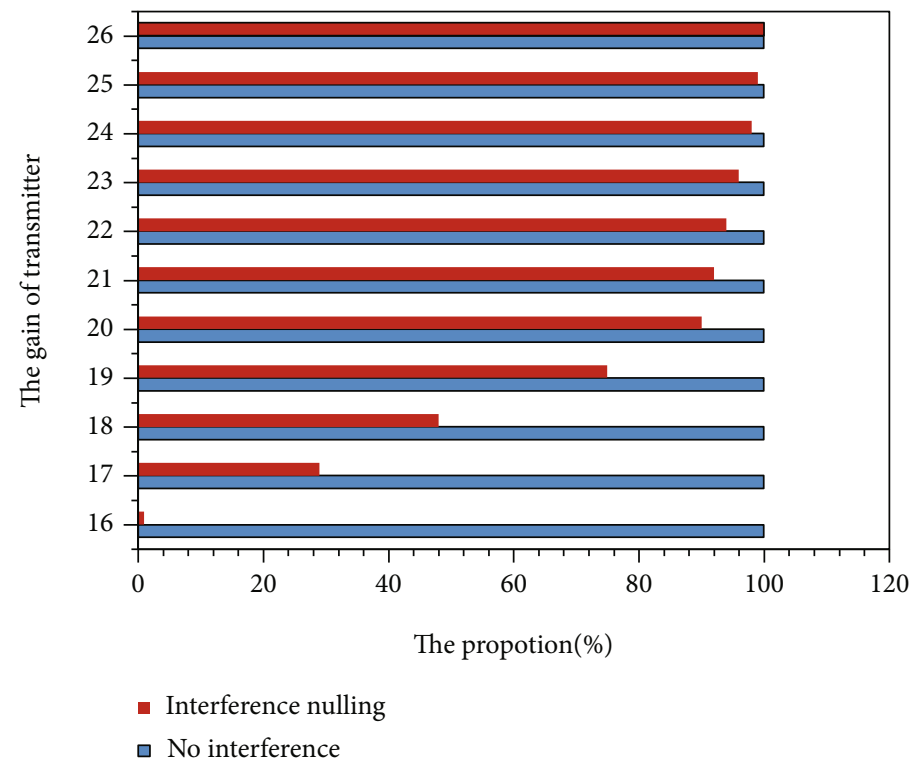

FIgURE 10: The success rate of Client1 receiving data packets from AP1 in different states of Client2.

the allocation of resources depends on the management system of these allocation activities, and the market pricing mechanism makes it difficult for relevant parties to complete the optimal allocation. Finally, individuals in one group will match multiple individuals in the other group. People in the other set also choose to match many people in the set. Compared with the previous two two-dimensional mapping models, the multidimensional two-dimensional matching theoretical model is more flexible and complex, and the analysis is more difficult. Therefore, there are still many problems to be solved in the research of the many-to-many reciprocity theory. Western countries have obtained some successful applications in the research of bilateral mapping theory earlier. For example, many matching problems such as the matching of practice and hospital selection, school selection of primary and middle school students, and match- ing of organ donation have all been effectively improved with the help of bilateral matching theory. Whether it is theoretical analysis or practical demonstration, using matching theory to optimize the design of matching methods can significantly improve matching performance and increase the satisfaction of the parties.

Many-to-one is widely used in life. After entering the university, the applicants complete their volunteers in the university and are finally accepted by the university. The job seekers choose to work in many companies and market units, professional athletes choose to join competitive clubs, and so on. These two-way matching situations are examples of one size fits all.

4.2. GPRS Module. When the wireless sensor network is running in the site or area where the gateway is located and the 


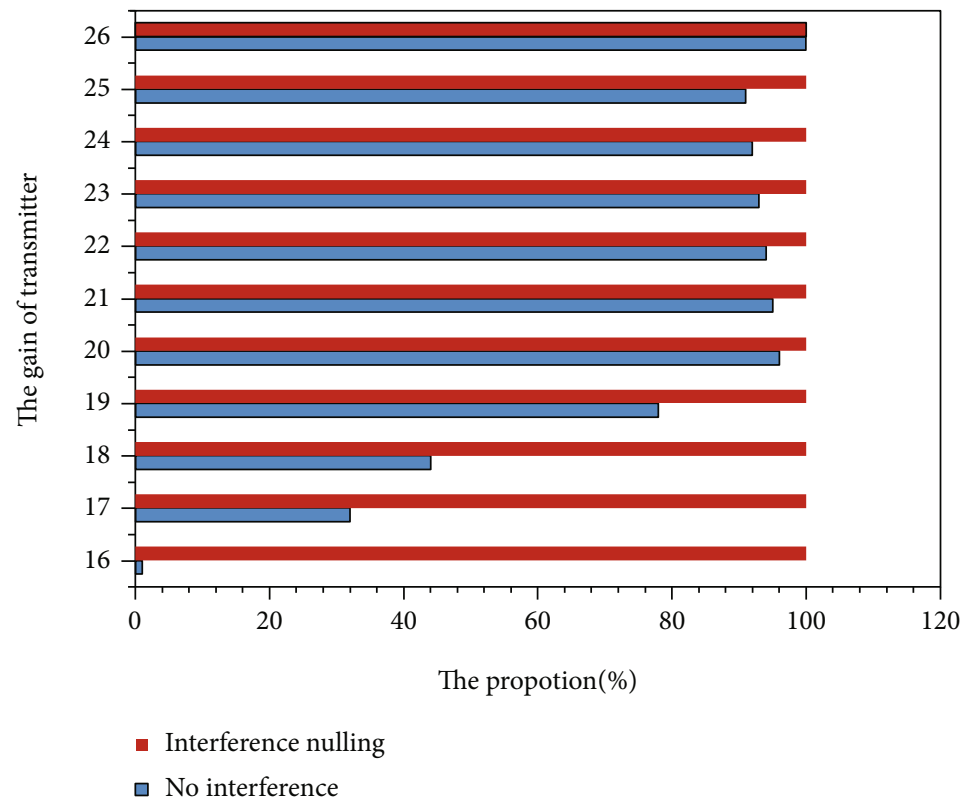

FIgURE 11: The success rate of AP1 receiving data packets from Client2 in different states.

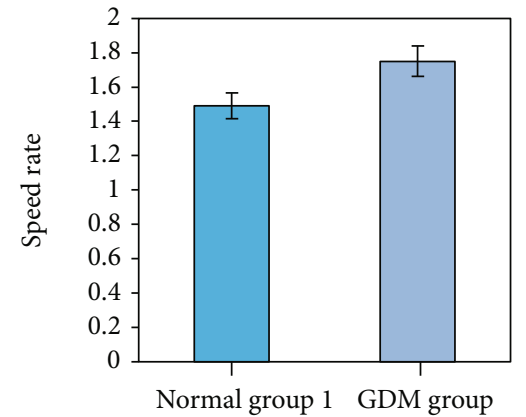

Type

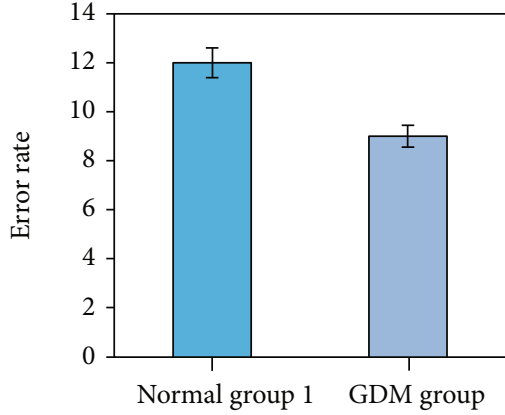

Type

Figure 12: Gain comparison of the two algorithms.

wired network cannot be deployed, long-distance wireless communication technology needs to be used to transmit data through the Internet through the Zigbee network. Although Zigbee is only a local area network with a limited coverage area, it can be connected to the existing mobile network, the Internet, and other communication networks, connecting many Zigbee LANs into a whole. With regard to effectively solving the problem of blind area coverage of mobile networks, we know that existing mobile networks have blind areas in many places, especially in the field such as in railways, highways, oil fields, and mines. The cost of adding a mobile base station or repeater is considerable. At this time, using the Zigbee network to cover the blind area not only is economical and effective but also is often the only feasible method now. In this process, the GPRS network can be used to transmit data from the lower layer of the Zigbee network to the control center. The Ocean Sense project introduced this method to send the natural environment data collected by the underlying network to the remote control center. Therefore, it is necessary to add a GPRS trans- mission function in the design and development of the portal to increase the scope of application of the portal.

GPRS stands for General Packet Radio Service, which transmits data through base stations covering a specific area, using the built-in GPRS deployment panel when designing the gateway for GPRS network mode. Considering factors such as power consumption, size, and price, this article uses the SIM800C chip produced by SIMCOM to transmit serial data through the GPRS network. SIM800C works in the four frequency bands of $850 / 900 / 1800 / 1900 \mathrm{MHz}$ and supports the $3.4 \mathrm{~V} \sim 4.4 \mathrm{~V}$ operating voltage. In addition, its control method is simple. We only need to send AT commands through the serial port of the single-chip microcomputer for the initial configuration of data transmission and reception. SIM800C integrates the TCP/IP protocol stack, and the TCP and UDP data transmission between the gateway and the control center can pass through this unit. The connection between the device and the gateway is also realized through an external interface. Because the Wi-Fi function and GPRS function of the gateway do not need to be used 
at the same time in actual use, the communication between the GPRS module and the gateway also uses serial port 3 in the actual design. Before the gateway sends data through the GPRS module, it must be prepared and configured. The preparation of the SIM800C GPRS module is mainly done by sending an AT command string through the STM32 serial port.

4.3. Vector Regression Method. In the process of human evolution and development, learning ability plays a vital role, by acquiring, analyzing, and summarizing known information; discovering certain laws and then predicting the failure process according to these laws; and directly obtaining information through observation. People also hope that computers can simulate human learning ability. This is a machine learning problem. Machine learning is a research hotspot in recent years - an important aspect of artificial intelligence. The significance of its research is to enable the computer to discover its inherent relevance by learning a large amount of data and to predict and judge future data.

The related theories of statistics have laid the foundation for the research of machine learning. The statistical theory (statistical learning theory or SLT) studied in this article is different from traditional statistics. This is a machine learning study that provides fewer data samples. Support vector machine (SVM) is the product of this theory, and its basic idea is to find a way to correctly classify different types of samples. Vector Regression Support (SVR) is a regression algorithm based on the idea of SVM, which has been successfully applied in practical engineering. The basic idea of the algorithm is to increase the dimensionality of the data and construct a decision function in a high-dimensional space to achieve the purpose of linear regression. In this process, the method of choosing regression parameters will determine whether its theoretical advantages can be realized. Density is actually an abbreviation; the full name is the degree of density. Is that not easier to understand? For example, what is commonly referred to as density refers specifically to mass density, that is, how dense the mass is. So naturally, the density of mass is the mass divided by the volume. The linear density is the density on a line, and the areal density is the density on a plane. Then, the density dimension is the density in three-dimensional space.

The ability of machine learning to correctly predict the data to be extracted through known data is a generalization of machine learning. Experience has shown that the best prediction results do not necessarily come from the slightest training error, which is a phenomenon of over-learning. This problem is when the sample is insufficient or the machine learning design is not logical enough and the two are related.

In order to minimize the true risk, the confidence interval must be reduced. The traditional method of adjusting the confidence interval is to select an appropriate learning model. The method given by statistical learning is to construct a series of function subsets arranged in the order of $\mathrm{VC}$ dimension. Among them, there must be a subset with the least empirical risk. In this way, the sum of the empirical risk and the confidence interval is the smallest. The idea is to minimize structural risk (SRM).
There are usually two ways to apply SRM: one is to find the minimum empirical risk of the function itself to get the function with the shortest confidence interval; the other is to transform its structural subgroup to select the function of the subset with the shortest confidence interval.

\section{Conclusions}

The experimental results show that the support vector regression method proposed in this paper based on the wireless network communication for medium and long-term predictions of regional economy, compared with the traditional support vector regression method, has better statistical effects, and the statistical indicators are more comprehensive. Both the retention rate and the stability of use have been improved. The article introduces related content about wireless network sensors and introduces the selection of sensors and the improvement of algorithms for space occupancy. The Zigbee wireless sensor network design was carried out. The transmitter transmits data under different gain conditions, and comparing the correct rate of Client1 successfully receiving data packets from AP1 when Client2 does not interfere with Client1 interferes with Client1 and when the interference is set to zero. The KMO value and Bartlett sphere tests were performed. The experimental data shows that the distance between AP1 and AP2 is $0.4 \mathrm{~m}$, and the distance between AP2 and Client2 is $0.6 \mathrm{~m}$. When BPSK is used for OFDM modulation, $2500 \mathrm{MHz}$ is used as the USRP center frequency, and $0.5 \mathrm{MHz}$ is used as the USRP bandwidth, AP1 can send data packets. The length is 100 bytes, the number of sent data packets is 100 , the gain of Client 2 is $0-38$, the receiving gain of AP2 is 0 , and the receiving gain of AP1 is 19. The shortcomings of this article are as follows: (1) The amount of data collected in the sample is relatively limited. In future research, the experimental sample can be expanded to obtain the credibility of the research results. (2) The vector regression algorithm designed in this paper does not have separate control variables in the process of simplifying the algorithm. Although the actual experimental results did not have much impact, the reliability of the algorithm should be more stringent in future research. (3) The article's introduction to algorithm comparison is not comprehensive enough, and no citations and explanations of other algorithms for comparison are added. In future experiments, relevant content can be added to make the article more reasonable.

\section{Data Availability}

No data were used to support this study.

\section{Conflicts of Interest}

The author states that this article has no conflict of interest.

\section{Acknowledgments}

This work was supported by the Research on Situational Awareness and Prevention of Financial Risks of Insurance 
Companies under the Background of Big Data Finance (2020RKB01556). This work was supported by the Situational Awareness of Financial Risks based on Deep Learning of Big Data (SKLCN-2020-06).

\section{References}

[1] A. Raza, S. Junaid Nawaz, S. Wyne, A. Ahmed, M. A. Javed, and M. N. Patwary, "Spatial modeling of interference in inter-vehicular communications for 3-D volumetric wireless networks," IEEE Access, vol. 8, no. 99, pp. 108281-108299, 2020.

[2] X. Tang and G. Wang, "Design and analysis of e-commerce and modern logistics for regional economic integration in wireless networks," EURASIP Journal on Wireless Communications and Networking, vol. 2020, no. 1, 2020.

[3] R. Bertini, H. Wang, T. Knudson, and K. Carstens, "Preparing a roadmap for connected vehicle/cooperative systems deployment scenarios: case study of the State of Oregon, USA," Transportation Research Procedia, vol. 15, no. 1, pp. 447458, 2016.

[4] B. R. Shrestha, K. D. Sagar, and K. R. Rao, “An optimized multipath routing for secure communication of wireless sensor network," International Journal of Recent Technology and Engineering, vol. 7, no. 6, pp. 1005-1010, 2021.

[5] V. Borisov and A. Pudalov, "Means of creating interference in wireless communication networks," Scientific Papers Collection of the Angarsk State Technical University, vol. 2020, no. 1, pp. 25-34, 2020.

[6] Q. Liu, X. Wang, and P. Li, "Interference signal separation algorithm in anonymous communication of wireless network for ships," Journal of Coastal Research, vol. 93, no. sp1, pp. 633-636, 2019.

[7] K. Purnima Sharma et al., "Evolution of mobile wireless communication networks (0G-8G)," International journal of electrical engineering and technology, vol. 6, no. 2, pp. 163-176, 2016.

[8] S. Liu, Y. Yang, and Y. Wang, "Integration of museum user behavior information based on wireless network," Mobile Information Systems, vol. 2021, no. 5, Article ID 6847144, 2021.

[9] Y. Zhao, M. Prabhu, R. R. Ahmed, and A. K. Sahu, "Research trends and performance of IIoT communication networkarchitectural layers of petrochemical industry 4.0 for coping with circular economy," Wireless Communications and Mobile Computing, vol. 12, no. 1, 2021.

[10] G. Lei, L. Ji, R. Ji, Y. Cao, X. Shao, and X. Huang, "Extracting low-rate DDoS attack characteristics: the case of multipath TCP-based communication networks," Wireless Communications and Mobile Computing, vol. 11, no. 4, 2021.

[11] L. Duan, K. Zhang, B. Cheng, and B. Ren, "Privacy threats of acoustic covert communication among smart mobile devices," Wireless Communications and Mobile Computing, vol. 2, no. 3, 2021.

[12] L. M. B. Ribeiro, I. Müller, and L. B. Becker, "Communication interface manager for improving performance of heterogeneous UAV networks," Sensors, vol. 1, no. 13, pp. 42-55, 2021.

[13] F. Zheng, Z. Pi, Z. Zhou, and K. Wang, "LEO satellite channel allocation scheme based on reinforcement learning," Mobile Information Systems, vol. 2020, no. 3, Article ID 8868888, 2020.
[14] S. Verma, M. Ghazel, and M. Berbineau, "Model-based dependability evaluation of a wireless communication system in a virtually coupled train set," IFAC-PapersOnLine, vol. 54, no. 2, pp. 179-186, 2021.

[15] G. Wang, "A multi-target tracking and detection algorithm for wireless sensor networks," International Journal of Circuits, vol. 15, no. 4, pp. 661-665, 2021.

[16] E. Myasnikova, A. Samsonova, M. Samsonova, and J Reinitz, "Support vector regression applied to the determination of the developmental age of a Drosophila embryo from its segmentation gene expression patterns," Journal of Superconductivity \& Novel Magnetism, vol. 29, no. 1, pp. 1641-1646, 2016.

[17] G. Santamaría-Bonfil, A. Reyes-Ballesteros, and C. Gershenson, "Wind speed forecasting for wind farms: a method based on support vector regression," Renewable Energy, vol. 85, no. 4, pp. 790-809, 2016.

[18] B. Wolff, J. Kühnert, E. Lorenz, O. Kramer, and D. Heinemann, "Comparing support vector regression for PV power forecasting to a physical modeling approach using measurement, numerical weather prediction, and cloud motion data," Solar Energy, vol. 135, no. 10, pp. 197-208, 2016.

[19] F. Kang and J. Li, “Artificial bee colony algorithm optimized support vector regression for system reliability analysis of slopes," Journal of Computing in Civil Engineering, vol. 30, no. 3, pp. 401-504, 2016.

[20] R. Khelif, B. Chebel-Morello, S. Malinowski, E. Laajili, F. Fnaiech, and N. Zerhouni, "Direct remaining useful life estimation based on support vector regression," IEEE Transactions on Industrial Electronics, vol. 64, no. 3, pp. 2276-2285, 2017.

[21] S. Chen, K. Jeong, and W. Härdle, "Support vector regression based GARCH model with application to forecasting volatility of financial returns," Social Science Electronic Publishing, vol. 92, no. 4, pp. 398-399, 2017.

[22] C. Tao and S. Lu, "Accurate and efficient traffic sign detection using discriminative AdaBoost and support vector regression," IEEE Transactions on Vehicular Technology, vol. 65, no. 6, pp. 4006-4015, 2016.

[23] J. Á. Muñiz, R. G. Ayestarán, J. Laviada, and F. Las-Heras, "Support vector regression for near-field multifocused antenna arrays considering mutual coupling," International Journal of Numerical Modelling Electronic Networks Devices \& Fields, vol. 29, no. 2, pp. 146-156, 2016.

[24] P. R. Filgueiras, N. A. Portela, S. Silva et al., "Determination of saturates, aromatics, and polars in crude oil by ${ }^{13} \mathrm{C}$ NMR and support vector regression with variable selection by genetic algorithm," Energy \& Fuels, vol. 30, no. 3, pp. 1972-1978, 2016.

[25] G. Francesco, G. Rudy, and D. M. Giovanni, “Support vector regression for rainfall-runoff modeling in urban drainage: a comparison with the EPA's storm water management model," Water, vol. 8, no. 3, pp. 69-72, 2016.

[26] Z. Cai, Z. He, X. Guan, and Y. Li, "Collective data-sanitization for preventing sensitive information inference attacks in social networks," IEEE Transactions on Dependable and Secure Computing., vol. 15, no. 4, pp. 577-590, 2018.

[27] Z. Cai and Z. He, “Trading private range counting over big IoT data," in 2019 IEEE 39th International Conference on Distributed Computing Systems (ICDCS), Dallas, TX, USA, 2019.

[28] Z. Xu and Z. Cai, "Privacy-preserved data sharing towards multiple parties in industrial IoTs," IEEE Journal on Selected Areas in Communications (JSAC)., vol. 38, no. 5, pp. 968979, 2020. 
[29] W. Sun, J. Wang, N. Zhang, and S. Yang, "Scalable implementation of hippocampal network on digital neuromorphic system towards brain-inspired intelligence," Applied Sciences, vol. 10, no. 8, p. 2857, 2020.

[30] Z. Lv, A. K. Singh, and J. Li, "Deep learning for security problems in 5G heterogeneous networks," IEEE Network, vol. 35, no. 2, pp. 67-73, 2021.

[31] Z. Lv, H. Song, P. Basanta-Val, A. Steed, and M. Jo, "Next-generation big data analytics: state of the art, challenges, and future research topics," IEEE Transactions on Industrial Informatics, vol. 13, no. 4, pp. 1891-1899, 2017.

[32] D. Chen, P. Wawrzynski, and Z. Lv, "Cyber security in smart cities: a review of deep learning-based applications and case studies," Sustainable Cities and Society, vol. 66, p. 102655, 2021. 